\title{
Perfil clínico de pacientes dermatológicos encaminhados para avaliação oftalmológica
}

\author{
Clinical profile of dermatological patients referred to ophthalmological evaluation
}

Luiza Duarte Pittol ${ }^{1}$ (1), Natália Brambilla Antoniolli1 (1), Rodrigo Pereira Duquia' ${ }^{10}$, Renan Bonamigo² (1), Manuel Augusto Pereira Vilela1 ${ }^{10}$

1 Universidade Federal de Ciências da Saúde de Porto Alegre, Porto Alegre, RS, Brasil. ${ }^{2}$ Santa Casa de Misericórdia de Porto Alegre, Porto Alegre, RS, Brasil.

\section{Descritores:}

Dermatopatias; Anormalidades do olho; Encaminhamento e consulta; Cegueira; Transtornos da visão

Keywords: Skin diseases; Eye abnormalities;

Referral and consultation; Blindness; Vision disorders

Recebido: $23 / 12 / 2020$

Aceito: 11/7/2021

Autor correspondente: Manuel Pereira Vilela Rua Félix da Cunha, 496 - Bairro Floresta CEP: 90570-001 - Porto Alegre, RS, Brasil E-mail: mapvilela@gmail.com

Instituição de realização do trabalho: Santa Casa de Misericórdia de Porto Alegre.

Fonte de auxílio à pesquisa: não financiado.

Conflitos de interesse: os autores declaram que não há conflitos de interesses.

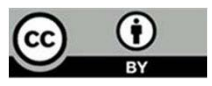

Copyright ()2021

\section{RESUMO}

Objetivo: Analisar o perfil clínico de pacientes em acompanhamento dermatológico encaminhados para avaliação oftalmológica.

Métodos: A amostra foi composta de pacientes dermatológicos encaminhados para avaliação oftalmológica, nos anos de 2016 e de 2017. Estudou-se a concomitância de doenças dermatológicas com as afecções oftalmológicas por meio da coleta simultânea do histórico dermatológico (prontuários registrados com dados gerais e diagnóstico) e de dados da consulta oftalmológica após o encaminhamento.

Resultados: Foram avaliados pela oftalmologia 224 pacientes, sendo $65 \%$ do sexo feminino, $80 \%$ caucasianos, com idade variando entre 1 mês e 85 anos. As situações cujo encaminhamento foi mais prevalente foram psoríase, lúpus, vitiligo e rosácea (18,3\%, 13,8\%, 12,9\% e 10,7\%, respectivamente). Fototerapia crônica e uso de hidroxicloroquina representaram 35,7\% e 22,3\% dos pacientes. Casos de neurofibromatose, micose fungoide, líquen plano, neoplasias de pele, atopias, pênfigo e esclerodermia também estiveram presentes. Cegueira legal foi detectada em $6 \%$, e deficiências visuais ligadas a afecções dermatológicas foram verificadas em $16,5 \%$ dos casos. As alterações oculares mais prevalentes foram catarata $(18,9 \%)$, blefarite $(15,9 \%)$, pterígio $(5,3 \%)$ e conjuntivite $(5,3 \%)$.

Conclusão: Encontrou-se elevada frequência de alterações oftalmológicas em uma população de pacientes com doenças dermatológicas. Assim, o estudo e a análise de manifestações oculares em pacientes dermatológicos podem auxiliar na detecção precoce e na prevenção de complicações.

\section{ABSTRACT}

Objective: To analyze the clinical profile of patients under dermatological care and referred to ophthalmological evaluation.

Methods: The sample comprised dermatology patients referred to ophthalmological evaluation in 2016 and 2017. The simultaneous occurrence of skin and ophthalmic diseases was studied, by collecting dermatological history (medical records containing general data and diagnosis) and ophthalmic consultation data following referral.

Results: A total of 224 patients were assessed, $65 \%$ were female, $80 \%$ were white, and age varied between one month and 85 years. The conditions more often referred were psoriasis, lupus, vitiligo and rosacea $(18.3 \%, 13.8 \%, 12.9 \%$ and $10.7 \%$, respectively). Chronic phototherapy and use of hydroxychloroquine were observed in $35.7 \%$ and $22.3 \%$ of patients, respectively. Cases of neurofibromatosis, mycosis fungoides, lichen planus, skin cancer, atopic dermatitis, pemphigus and scleroderma were also reported. Legal blindness was detected in $6 \%$ and visual impairment related to skin conditions in $16.5 \%$ of patients. The most prevalent ocular changes were cataracts (18.9\%), blepharitis (15.9\%), pterygium (5.3\%) and conjunctivitis (5.3\%)

Conclusion: A high frequency of ophthalmic changes in a population of dermatological patients was found. In this context, studying and analyzing ocular manifestations in dermatological patients could be useful in early detection and prevention of complications. 


\section{INTRODUÇÃO}

Doenças de pele inflamatórias crônicas e autoimunes, como psoríase, lúpus eritematoso, vitiligo e rosácea, são muito prevalentes e apresentam grande impacto na qualidade de vida, no bem-estar e na autoestima. Essas doenças frequentemente cursam com acometimentos extra cutâneos, destacando-se o ocular, o qual leva a uma demanda cada vez maior do serviço oftalmológico para acompanhamento e manejo ${ }^{(1)}$.

Segundo dados da Sociedade Brasileira de Dermatologia (SBD), a psoríase acomete aproximadamente 3\% da população mundial e 1,3\% da população brasileira, com incidência crescente. Trata-se de uma doença inflamatória crônica e cíclica, idiopática, manifestando-se preferencialmente como placas secas e avermelhadas, com escamas prateadas e esbranquiçadas. Em relação às comorbidades oculares, embora não existam muitos relatos na literatura atual, a prevalência estimada de danos oculares inflamatórios é de aproximadamente $10 \%$, sendo a maioria naqueles com artrite psoriática concomitante. Em estudo prévio, dentre cem pacientes do grupo caso com psoríase, os achados oculares estavam presentes em 58\%; já nos cem pacientes controles saudáveis, as manifestações estavam presentes em apenas $25 \%{ }^{(2)}$ As alterações mais frequentes foram a blefarite (39\%), o envolvimento corneano (16\%) e a conjuntivite $(14 \%)$.

A rosácea, doença vascular inflamatória crônica, ocorre em 1,5\% a 10\% das populações estudadas, sendo mais prevalente na faixa dos 30 aos 50 anos e preferencialmente em mulheres, segundo dados da Sociedade Brasileira de Dermatologia(3). O distúrbio se manifesta com quatro tipos clássicos de acometimento: eritêmato-telangiectásica, papulopustuloso, fimatoso/rinofima e ocular. Frequentemente, há olho seco e aumento do risco de blefarite, ainda segundo a Sociedade Brasileira de Dermatologia ${ }^{(3)}$. Embora seja uma doença dermatológica, em $58 \%$ a $72 \%$ dos pacientes os olhos estão acometidos com inflamação palpebral e da superfície ocular; um terço apresenta envolvimento corneano, podendo cursar com baixa visual significativa pela ulceração epitelial, irregularidades da superfície e cicatrizes na córnea. ${ }^{(4)}$ Outros achados comuns são blefarite, disfunção das glândulas de Meibomius, conjuntivite crônica e reações papilares crônicas, queixas de sensação de corpo estranho, secura, fotofobia e lacrimejamento.

O vitiligo, doença idiopática caracterizada por lesões cutâneas hipopigmentadas, acomete aproximadamente $0,5 \%$ da população mundial. Em estudo prévio com 45 pacientes com vitiligo cutâneo, dez apresentavam alterações oftalmológicas em regiões da íris, nervo óptico e epitélio pigmentar, com manifestações de hipopigmentação focal ou difusa. Apenas pacientes com manchas despigmentadas periorbitais mostraram essa associação. As manchas foram observadas bilateralmente em quatro dos pacientes acometidos e unilateralmente em seis deles. Nenhum paciente sem manchas apresentou dano ocular. ${ }^{15}$

O lúpus eritematoso sistêmico é uma doença crônica imunomediada, multissistêmica, que acomete preferencialmente mulheres (90\%) e negros. Classicamente, manifesta-se como dor articular, febre e rash em mulheres pós-puberais. As manifestações cutâneas estão presentes em $70 \%$ a $80 \%$, sendo característicos o rash malar, o lúpus discoide, a fotossensibilidade e as lesões na mucosa. Metade dos pacientes apresentam manifestação ocular, como lesão cutânea palpebral (lúpus discoide), síndrome de Sjögren (20\%), esclerites, lesões neuro-oftalmológicas (paralisia de pares cranianos, neuropatia óptica, lesões retroquiasmáticas e do córtex, uveíte, e vasculopatia retiniana). ${ }^{(1)}$ As alterações fundoscópicas podem servir como importantes marcadores de atividade da doença (expondo focos de exsudação algodonosa (isquemia) e micro-hemorragias; oclusões vasculares (artéria ou veia) e coroidopatia - isquemia e focos de descolamento do epitélio pigmentar).

Outras condições dermatológicas que cursam com acometimento oftalmológico são neurofibroblastoma, micose fungoide, dermatite atópica e pênfigo. Pacientes com dermatite atópica podem desenvolver ceratocone, cicatrização corneana ou conjuntiva e secreção de muco. Outra manifestação possível é a catarata atópica, começando com opacificação subcapsular do cristalino, geralmente bilateral e primariamente vista em adolescentes e jovens adultos. ${ }^{(6)}$

A alta prevalência dessas condições cutâneas na população brasileira e o aumento da demanda e dos encaminhamentos desses pacientes para os médicos oftalmologistas motivaram este estudo.

O objetivo do presente estudo foi analisar o perfil clínico de pacientes em acompanhamento dermatológico encaminhados para avaliação oftalmológica.

\section{MÉTODOS}

Realizou-se um estudo transversal por meio da coleta de dados em prontuários médicos para estimar a prevalência de alterações oftalmológicas em uma população de pacientes com doenças de pele e presença de queixas 
oculares, em um ambulatório terciário de Porto Alegre (RS). Os achados do exame oftalmológico foram analisados. Todos os pacientes foram submetidos à medida de acuidade visual (tabela de Snellen), refratometria, biomicroscopia, avaliação da resposta pupilar e da motricidade ocular, tonometria e fundoscopia binocular indireta. Os pacientes do Sistema Único de Saúde (SUS) foram encaminhados do Serviço de Dermatologia situado na Unidade Básica de Saúde Santa Marta, também em Porto Alegre, para o ambulatório de Oftalmologia da Universidade Federal de Ciências da Saúde de Porto Alegre.

A amostragem foi selecionada sistematicamente $e$ composta de pacientes em atendimento dermatológico e com queixas oculares, encaminhados ao atendimento oftalmológico no período descrito. A amostra não correspondeu toda a população analisada no ambulatório dermatológico, mas os referidos por queixas oftalmológicas. Os critérios de exclusão do estudo foram a falta de dados necessários no prontuário (considerando-se apenas os dados que seriam coletados no estudo) e os erros refrativos leves (miopia <-5, 0 , hipermetropia entre $+0,5$ e 2,0 e astigmatismo $<1,50$ ) ou corrigidos com boa resposta funcional. Não ocorreram casos excluídos, posto que o preenchimento foi feito no mesmo momento do atendimento, pelo examinador sênior. Erros leves foram excluídos para evitar a inclusão de casos meramente refrativos, que aproveitaram o interrogatório dermatológico para conseguir uma consulta oftalmológica, cuja demanda reprimida é elevada no estado do Rio Grande do Sul. A coleta de dados no ambulatório foi realizada no período de 2016 a 2017.

Todos os casos foram submetidos à mesma rotina de avaliação. Dados de identificação, idade, sexo, cor, profissão, procedência, medicacões em uso e queixas oftalmológica foram tabulados em planilha. O exame consistiu na medida da acuidade visual segundo a tabela de Snellen, com e sem correção, refratometria, avaliação da motricidade extrínseca, exame pupilar (direto, consensual e acomodativo), biomicroscopia, perimetria de confrontação, tonometria e fundoscopia sob midriase (lentes de não contato e oftalmoscopia indireta binocular). As imagens do segmento anterior e posterior foram obtidas com adaptadores para smartphones (em lâmpada de fenda e no exame fundoscópico com lente de 20 dioptrias - D). Todas as informações foram igualmente tabuladas. Os dados foram organizados em planilhas, e buscaram-se associações entre doenças dermatológicas e alterações oftalmológicas e suas repercussões anatomofuncionais. A associação foi por meio de percentual simples, uma vez que se trata de uma análise descritiva simples.

A variável dependente do estudo "alterações oculares" variou desde olho seco, inflamação ou infecção das estruturas oculares, fotofobia, alterações das estruturas anatômicas até casos mais graves de deficiência visual ou cegueira legal, a qual é definida no Brasil como acuidade visual igual ou menor que 0,05 (20/400) no melhor olho, com a melhor correção óptica.

O estudo obteve aprovação dos Comitês de Ética do Hospital Santa Marta (da Prefeitura de Porto Alegre) e da Universidade Federal de Ciências da Saúde de Porto Alegre (UFCSPA).

\section{RESULTADOS}

A maior parte dos 224 pacientes era do sexo feminino (65\%) e caucasiana (80\%). A média de idade foi 47 anos, com faixa etária variando de 1 mês a 85 anos (Tabela 1).

Dentre os pacientes que foram encaminhados para o serviço de oftalmologia encontraram-se alterações no exame oftalmológico em 169 pacientes, correspondendo a $75,44 \%$ da amostra estudada (Figura 1). Dentre as alterações encontradas, destacaram-se catarata $(n=34)$, blefarite $(n=27)$, pterígio $(n=9)$, conjuntivite $(n=9)$, disfunção das glândulas de Meibomius, nódulo de Lisch e

Tabela 1. Características demográficas da amostra populacional

\begin{tabular}{|c|c|c|c|c|c|c|c|c|c|}
\hline Característica & População total & $\begin{array}{c}\text { Pacientes com } \\
\text { psoríase }\end{array}$ & $\begin{array}{l}\text { Pacientes com } \\
\text { psoríase e } \\
\text { acometimento } \\
\text { ocular }\end{array}$ & $\begin{array}{l}\text { Pacientes com } \\
\text { lúpus }\end{array}$ & $\begin{array}{c}\text { Pacientes } \\
\text { com lúpus e } \\
\text { acometimento } \\
\text { ocular }\end{array}$ & $\begin{array}{l}\text { Pacientes com } \\
\text { rosácea }\end{array}$ & $\begin{array}{c}\text { Pacientes } \\
\text { com rosácea e } \\
\text { acometimento } \\
\text { ocular }\end{array}$ & $\begin{array}{c}\text { Paciente com } \\
\text { vitiligo }\end{array}$ & $\begin{array}{l}\text { Paciente com } \\
\text { vitiligo e } \\
\text { acometimento } \\
\text { ocular }\end{array}$ \\
\hline Idade, média & 47,6 & 48,8 & 56 & 45,5 & 58 & 46,8 & 44 & 44,4 & 54 \\
\hline \multicolumn{10}{|l|}{ Sexo } \\
\hline Masculino & 78 & 16 & 8 & 5 & 0 & 9 & 8 & 13 & 7 \\
\hline Feminino & 146 & 25 & 14 & 26 & 14 & 15 & 15 & 16 & 9 \\
\hline \multicolumn{10}{|l|}{ Etnia } \\
\hline Caucasianos & 179 & 34 & 19 & 26 & 10 & 21 & 20 & 24 & 14 \\
\hline Afrodescendentes & 41 & 7 & 3 & 5 & 4 & 3 & 3 & 5 & 2 \\
\hline Pardos & 2 & 0 & 0 & 0 & 0 & 0 & 0 & 0 & 0 \\
\hline Orientais & 2 & 0 & 0 & 0 & 0 & 0 & 0 & 0 & 0 \\
\hline Total & 224 & 41 & 22 & 31 & 14 & 24 & 23 & 29 & 16 \\
\hline
\end{tabular}


alterações de fundo de olho, como assimetria de escavações, escavações amplas e alterações de pigmentação (Figura 2). Os diagnósticos dermatológicos que estiveram mais associados a presença de alterações oftalmológicas foram: psoríase, lúpus eritematoso, vitiligo, rosácea, neurofibromatose, micose fungoide, líquen plano, neoplasia de pele, dermatite atópica, pênfigo e esclerodermia.

Pacientes com psoríase, lúpus eritematoso, vitiligo e rosácea foram os grupos em que ocorreram mais encaminhamentos, representando, respectivamente, 18,3\%,

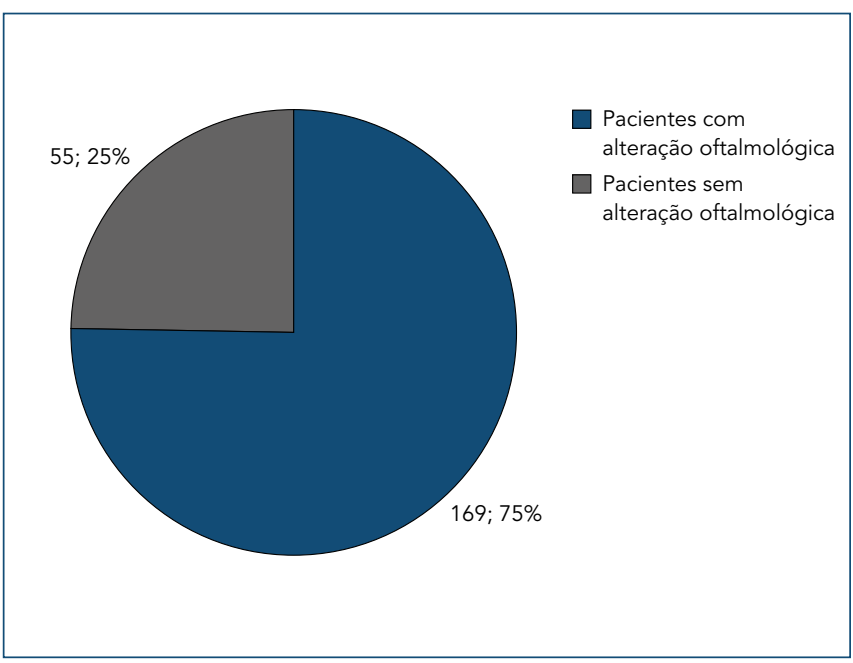

Figura 1. Frequência de alterações oftalmológicas.
13,83\%, 12,94\% e 10,71\%. Dentre esses pacientes, a maior prevalência de alterações oculares foi encontrada em pacientes com rosácea (95,83\% dos pacientes encaminhados com diagnóstico de rosácea apresentaram alteração oftalmológica, com destaque para a blefarite). Psoríase, lúpus eritematoso e vitiligo associaram-se a alterações oculares em 54\%, 45,16\% e 55,17\% dos pacientes estudados, respectivamente (Figura 3).

Dentre os pacientes com alteração oftalmológica, foram encontrados casos de cegueira legal e deficiência visual, correspondendo a $6 \%$ e a $16,5 \%$ dos pacientes encaminhados para o serviço de oftalmologia. Dentre os pacientes com deficiência visual, apenas três casos apresentaram associação com ametropias, sendo dois com miopias com mais de -5,OD e um caso de anisometropia e ambliopia secundária. As causas das deficiências visuais mais graves estiveram relacionadas com a dermatopatia ou seu tratamento (toxicidade da cloroquina, albinismo e glaucoma cortisônico), outras situações sistêmicas (sequelas de AIDS, uveíte por toxoplasmose, retinopatia diabética e vasculite retiniana) ou alterações primárias oculares não ligadas à dermatopatia (maculopatia por miopia e leucoma por úlcera córnea). Em relação a terapias dermatológicas, 80 pacientes realizavam ou já tinham realizado fototerapia (35,71\%), e 50 pacientes tratavam ou tinham histórico de tratamento com hidroxicloroquina $(22,32 \%)$.

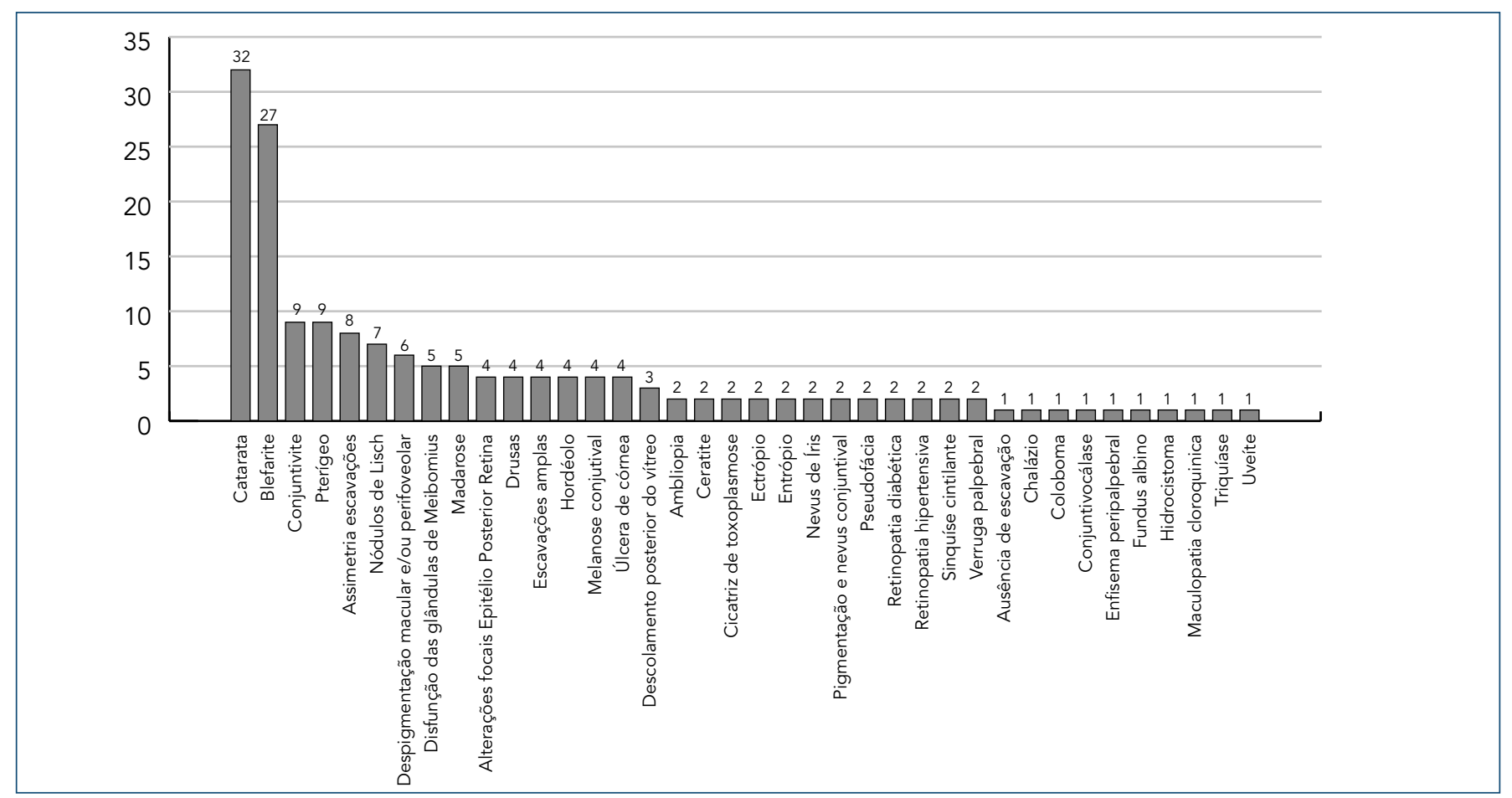

Figura 2. Incidência das alterações oftalmológicas. 


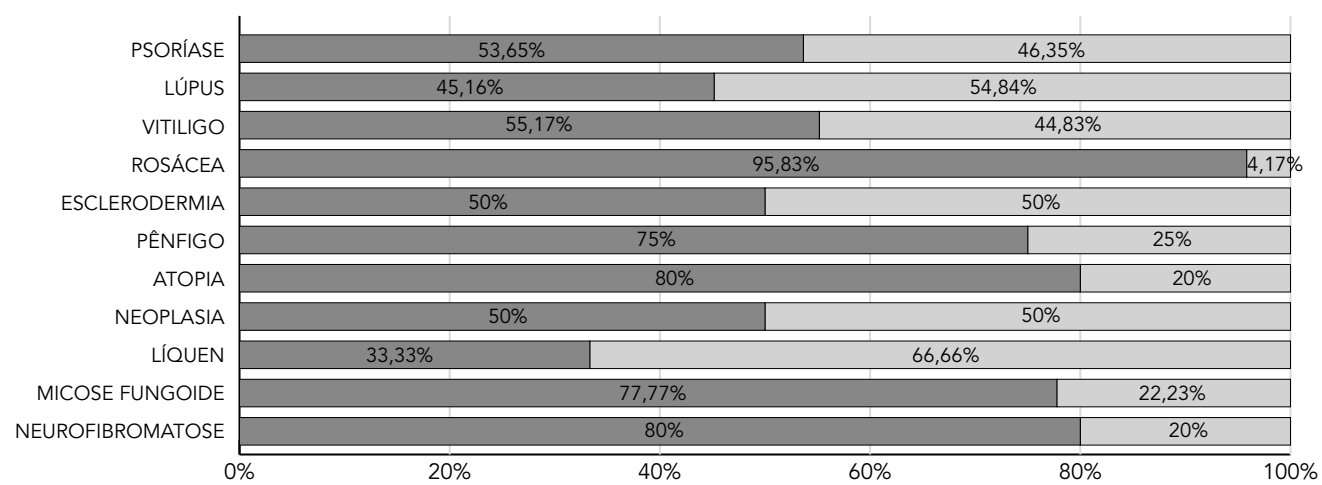

Figura 3. Doenças dermatológicas e alterações oftalmológicas.

\section{DISCUSSÃO}

Dentre o total de 224 pacientes analisados neste estudo, 169 apresentaram algum tipo de alteração oftalmológica, representando $75,44 \%$ da amostra. Nos grupos, a maior prevalência de associação entre os achados dermatológicos e oculares ocorreu na psoríase, no lúpus, no vitiligo e na rosácea. Os casos de cegueira legal permaneceram restritos a uma parcela menor da amostra (apenas 6\% dos casos), enquanto a deficiência visual esteve presente em $16,5 \%$ dos pacientes.

A psoríase apresentou associação significativa com achados oculares tanto neste estudo quanto em outros registros da literatura $^{(2)}$. Neste trabalho, foi a condição dermatológica de maior repercussão ocular, representando $24,26 \%$ do total de pacientes em que os achados oculares foram encontrados. Dos 41 pacientes da amostra, 22 apresentaram algum tipo de achado oftalmológico (53,65\%). A frequência das alterações oculares mais prevalentes neste estudo foi: oito pacientes com catarata, cinco com blefarite, dois com conjuntivite e nenhum com pterígio. As alterações estiveram mais presentes no sexo feminino e na média de idade de 56 anos. Kilic et al. ${ }^{(2)}$ mostraram que cerca de 60\% dos pacientes com a doença de pele apresentaram alguma alteração ocular quando comparados aos controles não doentes, nos quais se encontraram alterações oculares em 25\% da amostra. De forma semelhante, Abaggani et al. (7) avaliaram 235 pacientes com diagnóstico confirmado de psoríase, detectando alterações oculares em 80\% dos pacientes com correlação positiva entre as variáveis tempo de doença, escore no Psoriasis Area Severity Index (PASI) e presença de achados oculares.

O lúpus eritematoso sistêmico foi a segunda doença com maior associação neste estudo. É forte a associação entre as lesões de pele e as alterações oculares ${ }^{(1)}$. Cerca de metade dos pacientes apresentam olho seco, lesões cutâneas palpebrais, danos neuro-oftalmológicos e achados patológicos no fundo de olho. Em estudo de coorte retrospectiva(8) ${ }^{(8)}$ com 98 pacientes com lúpus eritematoso sistêmico, 29 tiveram envolvimento ocular (29,6\%), e em 20 deles houve mais de uma manifestação. No presente estudo, dos 31 pacientes com lúpus, 45\% apresentaram acometimento ocular, sendo pacientes com catarata, um com blefarite e um com pterígio. Como características demográficas, todos os pacientes afetados pertenciam ao sexo feminino, com média de idade de 58 anos. Estes achados estão de acordo com a literatura, demonstrando que, apesar das alterações oculares serem muito frequentes, são bastante diversas em suas possibilidades ${ }^{(1)}$.

De forma análoga à psoríase e ao lúpus eritematoso sistêmico, a literatura prévia já relatava associação entre vitiligo e rosácea e achados oculares. Em relação ao vitiligo, Albert et al.' (g) destacam diversas alterações oculares relacionadas, como despigmentação da retina e coroide, a qual esteve presente em 39\% dos pacientes com vitiligo. Neste vigente, entre a amostra de 29 pacientes com vitiligo, mais da metade apresentou envolvimento ocular $(n=16)$, sendo que cinco apresentaram catarata, três pterígio e um blefarite. Os acometidos eram predominantemente mulheres, sendo a faixa etária mais acometida a da quinta e a da sexta década de vida, com média de idade de 54 anos. Quanto à rosácea, a revisão de Vieira et al.' (') afirma que os olhos podem estar acometidos em 58\% a $72 \%$ dos casos, e o olho seco com teste de Schirmer anormal ocorre em mais de 50\% dos pacientes. Dos 24 pacientes com rosácea nesta amostra, a maioria dos pacientes apresentou alteração oftalmológica associada (95,8\%): três apresentaram catarata, quatro pterígio, dois conjuntivite, e a blefarite teve destaque, com 12 pacientes acometidos; novamente houve 
predominância do sexo feminino, e a média de idade foi de 44 anos. Apesar dos números encontrados nesta pesquisa serem significativamente menores em relação a essas literaturas ' 4', ainda assim vitiligo e rosácea estiveram entre as doenças mais relacionadas ao acometimento ocular no presente estudo.

Nesse estudo, as principais limitações foram as inerentes a estudos observacionais: possibilidades de viés de seleção, viés de aferição, envolvimento de fatores de confusão e achados ocasionais na análise. A amostra populacional atingida pode ser considerada um viés importante, pois abrangeu apenas os pacientes com queixas oculares encaminhados ao serviço de oftalmologia no período de 1 ano. Entretanto, os resultados encontrados no presente estudo apresentam dados semelhantes e que são confirmados por outros estudos citados nos parágrafos acima (1:;2;;7;:;;9), os quais já associavam alterações oculares com doenças de pele. Afasta-se, assim, a possibilidade de que a amostragem não seja válida ou representativa da população. Outra limitação a ser enfatizada foi o fato de as duas medidas (achados oftalmológicos e doenças de pele) terem sido realizadas ao mesmo tempo, característica de um estudo de prevalência. Assim, torna-se impossível estabelecer uma relação causal entre as duas variáveis. Não há como saber se as manifestações oculares antecederam ou foram consequência das doenças dermatológicas. Contudo, o delineamento transversal foi usado em função de critérios logísticos e orçamentários.

Como fortalezas do estudo, destaca-se o método segundo qual se investigou a presença de alterações oculares em um serviço especializado em oftalmologia e com base em exame físico oftalmológico completo. Outro destaque foi a geração de hipóteses que o estudo observacional permite, servindo como base para novos estudos, que busquem comprovar associação causal direta. Ademais, trata-se de um estudo de realização rápida, barato e de fácil reprodução.

\section{CONCLUSÃO}

Foi elevada a frequência de alterações oftalmológicas em uma população de pacientes com doenças dermatológicas. Assim, os estudos das alterações oftalmológicas em pacientes com doenças crônicas ou agudas de pele permitem a detecção precoce e o acompanhamento dessas complicações, prevenindo consequências mais graves ou a evolução para deficiência visual e cegueira legal e, por conseguinte, melhorando a qualidade de vida e o bem-estar.

\section{REFERÊNCIAS}

1. Vilela MA, Colossi CG. Oftalmologia - Fundamentos para Graduação em Medicina. Apple Books, 2016

2. Kilic B, Dogan U, Parlak AH, Goksugur N, Polat M, Serin D, et al. Ocular findings in patients with psoriasis. Int J Dermatol. 2013;52(5):554-9.

3. Bolognia JL, Jorizzo JL, Schaffer JV. Dermatologia - SBD - $3^{\text {a }}$ edição. Apple Books, 2015.

4. Vieira AC, Höfling-Lima AL, Mannis MJ. Ocular rosacea--a review. Arq Bras Oftalmol. 2012;75(5):363-9

5. Bulbul Baskan E, Baykara M, Ercan I, Tunali S, Yucel A. Vitiligo and ocular findings: a study on possible associations. J Eur Acad Dermatol Venereol. 2006;20(7):829-33.

6. Mondzelewski L, Hagan C, White A. An 18-year-old male with severe bilateral cataracts and atopic dermatitis--a case report and review of the literature. Pediatr Dermatol. 2009;26(5):583-6.

7. Abbagani S, Kamath YS, Nayak S. A study on ocular morbidity among patients with psoriasis visiting a tertiary care hospital in Karnataka, Southern India. Ocul Immunol Inflamm. 2019;27(4):531-4

8. Dammacco R, Procaccio P, Racanelli V, Vacca A, Dammacco F. Ocular involvement in systemic lupus erythematosus: the experience of two tertiary referral centers. Ocul Immunol Inflamm. 2018;26(8):1154-65.

9. Albert DM, Nordlund JJ, Lerner AB. Ocular abnormalities occurring with vitiligo. Ophthalmology. 1979;86(6):1145-60. 